\title{
THE CONDITION AND MANAGEMENT
}

\author{
OF THE

\section{INTESTINE AFTER ABDOMINAL SECTION}

\author{
CONSIDERED IN THE LIGHT OF PHYSIOLOGICAL \\ FACTS.
}

BY

JOHN D. MALCOLM, M.B., C.M., F.R.C.S.EdIN., ASSISTANT SURGEON TO THE SAMARITAN FREE HOSPITAL.

Received January 21st-Read Oct. 25th, 1887.

A KNOWLEDGE of the nervous mechanism of the intestine is important to the comprehension of some of the phenomena which may be induced by an abdominal section. As a preliminary to the study of these phenomena, I propose, therefore, very briefly to direct attention to certain facts connected with its nervous supply which seem to me to have a direct bearing on the condition of the intestine after it has been interfered with during an operation.

The nerves of the intestine are mainly derived from the plexuses of the sympathetic which accompany the branches of the mesenteric arteries. ${ }^{1}$ In the wall of the bowel these nerves terminate in two plexuses, one of which (the plexus myentericus of Auerbach) distributes its fibres to the muscular coat of the gut, while the other (Meissner's

1 Turner's 'Introduction to Anatomy,' pp. 711 and 723. 
plexus) gives branches "to the muscularis mucosø, the smooth muscular fibres of the villi, and the glands of the intestine." There is evidence that the nerves of the bowel are in intimate physiological connection with the central nervous system. ${ }^{2}$ But the plexuses in the wall of the gut possess also, and in a very high degree, an automatic power comparable to that of the ganglion-cells of the heart. This automatic power enables the intestine even "when cut out of the body to execute, apparently spontaneously, movements for some time;"s and during life it plays an important part in the performance of all the functions of the gut. 'These functions are therefore readily influenced by local stimuli, which may be chemical, mechanical, thermal, or electric in their nature, and which may act from within the bowel or from without. Or the stimulus may consist in some condition of the blood passing through the intestinal vessels.

The effect of stimulation of Auerbach's plexus is first to increase peristalsis, which may become violent, spasmodic, and painful. But if the stimulus be sufficiently severe or prolonged, paresis or complete paralysis of the muscular power of the gut results. The condition of the blood flowing through the intestinal capillaries has a most important influence on the intestinal movements. Marked irritability, followed by paralysis of the intestine, may be induced by congestion or plugging of its vessels; and the spontaneous evacuation of the bowel which sometimes occurs on the approach of death is attributable to vascular changes in its wall. Inflammation too may be accompanied by irritability of the muscular wall of the bowel, as is frequently observed in catarrh of its mucous membrane, while severe peritonitis produces absolute muscular paralysis of the gut."

The secretion of the digestive juices is also under the

1 Stirling's translation of Landois' ' Physiology,' 2nd edit., p. 337.

2 Ibid., p. 350.

3 Ibid., p. 337.

+ Ibld., p. 337, et seq. 
control of the nervous system, and it has been shown that division of the nerves distributed to the gut is followed by a copious flow of watery secretions from the intestinal glands $;^{1}$ but neither the influence of the various nerves and ganglia on the secreting structures nor the extent to which the glands are capable of independent action is well determined. Nor is the effect of nervous conditions on the development of gases in the intestine well understood. The production of these gases has been attributed to processes of fermentation, to the exchange of gases in the intestine for those in the blood, and to excessive swallowing of air; but these processes do not seem sufficient to account for the large quantities of gas which are developed in the intestines in certain nervous diseases. Simple transfusion ${ }^{2}$ and secretion ${ }^{3}$ of gases by the mucous membrane have been offered as explanations, but neither has been proved to take place to any great extent. ${ }^{4}$ It seems to me that an excessive development of gases in the intestine may sometimes be produced by a simple chemical action of the secretion (it may be an altered secretion) from one gland, or set of glands, on that from another. In Stirling's translation of Landois' 'Physiology' it is stated $^{5}$ that carbonic acid is set free in the stomach on the contact of the saliva, which contains carbonates, with the acid of the gastric juice. But the pancreatic, biliary, and intestinal secretions also contain a considerable percentage of carbonic acid in the form of carbonates. ${ }^{6}$ In the gastric juice during healthy digestion "the amount of free acidity is, however, slight, because the hydrochloric acid which the gastric juice contains combines for the time being with pepsin and proteids, forming a

1 Stirling's translation of Landois' ' Physiology,' 2nd edit., p. 397.

2 Foster's ' Physiology,' 3rd edit., p. 278.

3 'American Journal of Medical Science,' N. S., iv, 403.

4 Kirke's ' Physiology,' 3rd edit., pp. 366, 367 ; Quain's ' Dict. of Medicine,' art. "Flatulency."

6 2nd edit., p. 359.

- Stirling's translation of Landois' 'Physiology,' 2nd edit., pp. 361, 383, 395. 
compound which does not give an acid reaction."l But we know that urine, sweat, and saliva may be secreted profusely under the influence of purely mental stimulations; and if a flow of gastric and intestinal juices should occur from similar influences or from other disordered conditions, when the stomach is empty or contains food which is unsuitable in quantity or in quality, then the gastric secretions may pass, unaltered, into the duodenum, and may there mix with the secretions from the pancreatic, biliary, and intestinal glands. Moreover, from observations by Reichman, " gastric juice appears sometimes to be secreted with a larger proportion of acid than normal.", A profuse flow of gastric juice, especially if thus rich in acid, in the absence of a normal object for its energies, would be expected, on mixing with the juices in the duodenum, to set free carbonic acid gas by the action of its acid on the carbonates which these juices contain. Other less understood chemical reactions may take place, and as there can be no doubt that nervous conditions do influence the amount and the quality of the secretions, it is conceivable that large quantities of gas might thus be produced almost with explosive suddenness; and this seems a possible explanation of the enormous development of flatus which takes place in certain conditions.

The exact sources of the gases in the alimentary canal, and the causes of their presence in excessive quantity, are much in need of elucidation; but there is no doubt that excessive flatulence "seems to be in some measure connected with defective or deranged innervation, for flatulent accumulations in the stomach and bowels, not attributable to any other cause, are frequent in nervous affections such as hysteria, hypochondriasis, and strong mental emotions."

It would appear therefore that all the functions of the intestine are under the control of the automatic nervous

\footnotetext{
1 Lauder Brunton's ‘ Diseases of Digestion,' p. 12.

2 Ibid., fontnote, p. 33.

3 Wood's ' Practice of Medicine,' vol. i, p. 713.
} 
plexuses in its walls; and that these plexuses, while closely associated with, and to a great extent controlled by, the central nervous systems, are readily affected by any disturbing influences which may act directly upon them.

Many disturbing influences are brought to bear on these local nervous centres in the wall of the gut whenever the surgeon interferes with the peritoneum. The disturbance may be very slight, as in the operation of tapping the peritoneum for ascites. On the other hand the intestines may be subjected to the most abnormal conditions and to much physical injury, as in the removal of an adherent tumour from within the peritoneal cavity. Or a septic agent may be introduced. In all cases the nervous plexuses of the intestine are more or less affected, their activity being first increased and afterwards paralysed in proportion to the severity and duration of the stimulus. A condition is produced comparable to that which gives rise to the after-constipation which frequently follows the administration of castor-oil and other purgatives, and which owns exactly the same cause,-exhaustion of the nervous centres in the wall of the intestine.

Theoretically, then, paresis, or paralysis of the gut, results from every abdominal section. Practically the effect is often demonstrable. When an abdomen is first opened the presenting coils may be seen in a state of active peristalsis, but after a short exposure they become limp and motionless, and for from an hour or two to some forty-eight hours or even longer after the operation there is no sign (except the vomiting due to the anæsthetic) of any intestinal activity. Flatus does not escape from the bowel, and there is no abdominal distension. After a variable time, however, recovery of function in the gut is shown, if the case do well, by the passage of flatus from the anus, when a tube is passed through the sphincter ani ; by freedom from abdominal distension and from vomiting; and, later, by the rectum becoming full of fæces or by the occurrence of an act of defæcation.

But though the paralysis does not continue, the mus- 
cular power of the intestine is diminished for some time after operation, and this diminution of power is greatly aggravated by the want of exercise. The constraint which is necessarily put on the position of the patient also hampers the power of the gut by impeding the use of the accessory muscles of defæcation for. the purpose of expelling flatus. The existence of temporary intestinal paresis at this time is shown by the facts that flatus may escape freely if a tube be passed through the anus, when if left to the efforts of nature it is retained by the sphincter ani; that when an obstruction does occur the intestines may, and generally do, distend without the least pain; and that the bowels almost always require some assistance by enema or by laxative medicine at their first movement.

It now and then but rarely happens that very little gas is generated in the intestine as a consequence of abdominal section; on the contrary, it is usual for a good deal to be evolved after the intestine begins to revive; and in that case, if the patient do well, flatus escapes freely every three or four hours when a tube is passed into the rectum to facilitate its exit. This free escape of flatus continues usually for three or four days after its commencement, and then the amount of gas which escapes gradually decreases until it is no longer noticeable. The amount of gas which may be generated is most obvious when for some reason it does not escape from the bowel. Under such circumstances immense abdominal distension may take place in a few hours. This excessive development of gases during the recovery of the intestine from the effects of operation seems to be " connected with defective or deranged innervation," and in this respect resembles the flatulency of hysterical and other nervous conditions.

But whatever the cause of the development of gases in the alimentary canal after abdominal section, their speedy escape, through the rectal tube, or by the efforts of nature, is one of the most important signs that a case of this kind is doing well. If flatus does not escape freely some 
condition may be presumed to exist which interferes with the passage of the contents of the alimentary canal, or with the peristaltic action of the gut. If distension of the abdomen or vomiting follow, this presumption becomes a certainty, and it is then of the greatest importance to discover, and if possible to remove, the condition which causes the retention of flatus.

This may be :

1. Paralysis of the muscular wall of the gut.

2. Some malposition of a portion of the gut, brought about by the disturbance and rearrangement of the abdominal contents during operation.

3. Blocking of the lumen of the intestine by fæces.

4. A malformation, congenital or acquired, acting alone or by aggravating some other condition.

5. The accumulation of serum or of blood and serum in the peritoneal cavity, or escape into that cavity of irritating matter of any kind.

6. Inflammation of the peritoneum covering the gut.

7. General septic peritonitis.

8. Some pathological state of the bowel, such as one of the common forms of hernia, an intussusception, and others, which may occur as a concomitant disease in no way brought about by the operation, and to which therefore I will not refer again.

Of these we have seen that the first-paralysis of the muscular wall of the gut-follows every abdominal section in proportion to the amount of the interference with the intestines. The paralysed condition, if no other complication occurs, usually passes off in a few hours, but there is evidence that the bowel is distinctly wanting in power for from four to six or more days after operation. This condition is a most important factor in the production of obstruction of the intestine after abdominal section. It is obvious that in this state the bowel must be prone to yield to, rather than to remove, any opposition to the downward passage of its contents, so that a very slight impediment, which would be of no consequence whaterer vol. LXXI. 
during health, may lead to the most disastrous results. It would even appear that at this time such natural obstacles as exist at the anus, and in the friction of the more solid fæces which accumulate in the rectum and colon, may be the cause of a fatal obstruction.

Mr. Lawson Tait has twice seen death from paralysis of the intestine a week after operation. He says : "Two of my fatal cases, where the ligature had been used, were due to a kind of paralysis which was perfectly inexplicable. Dr. Batty tells me he has seen the same thing. In both instances the patients had made satisfactory progress until the sixth day after operation, without the slightest interruption, when suddenly the abdomen became greatly distended, incessant vomiting occurred, and the patient rapidly sank. After death nothing could be found except enormous distension of the abdomen by fluid fæces and gases. In both cases temporary relief was produced by tapping the intestines. The only explanation I can offer of the fatality in these cases is that some mysterious influence, similar perhaps to that which causes tetanus, brought about this unexpected and inexplicable end."1

I have met with a very similar case. Ovariotomy had been performed, and the patient progressed favorably until the seventh night after operation. There was then no cause for alarm when I saw her at 6.30 p.m. Vomiting came on about four hours after this, but the nurse did not send for assistance, and the patient vomited a great deal all night. In the morning, between 6 and 7 o'clock, the bowels moved spontaneously, and a large motion was then brought away by enema. Vomiting immediately ceased and did not return, but at 7 a.m. the temperature was $99.8^{\circ}$ F.- the highest that had been recorded since the first night after operation. At 9 $o^{\prime}$ clock the temperature was $101 \cdot 2^{\circ}$, and at the morning visit, at 9.30 , the abdomen was immensely distended, while the pulse. was 120 and extremely feeble. The temperature continued to rise, and the patient died on

1 Tait's 'Diseases of the Ovaries,' 4th edit., p. 309. 
the afternoon of the same day. At the necropsy I found that the large intestine was very small and had very thick walls, and that there was a piece of small gut adherent to the brim of the pelvis. The small intestine was greatly distended by fluid fæces and gases. The stomach and duodenum were also enormously dilated, and the former had evidently been chronically enlarged. The peritoneum was congested, but there was no visible lymph anywhere, no fluid in Douglas's pouch, and no sticking of peritoneal surfaces to each other, except where the parts had been divided. The explanation of this death seems to be that owing to the contracted condition of the colon and the chronic dilatation of the upper part of the alimentary canal, the muscular power of the gut was placed at a disadvantage. In spite of this the intestine was recovering from the paresis which followed the operation, and the small bowel was able to propel its fluid contents downward into the colon. But when it became necessary to remove the more solid matter which had accumulated in the large gut, the exhausted and embarrassed intestine was unequal to the task. Regurgitation with symptoms of obstruction resulted, and the repeated acts of vomiting produced sufficient stimulation and succussion to move the bowels. When these were well cleared the vomiting ceased; but so much exhaustion had been induced that, before the evacuation of the bowels occurred, the temperature had begun to rise and the patient could not be saved. Had the intestinal peristalsis been aided by an enema, or by a gentle laxative, whenever the vomiting began, or before this, I think that the issue of the case might have been different.

I have made more than one post-mortem examination at the Samaritan Free Hospital, in which the cause of the retention of flatus was not apparent, but in which intestinal distension appeared to be kept up by the pressure of a distended coil on a lower part of the gut. In other cases $I$ have found considerable lengths of small intestine decidedly narrower than the small gut generally. In 
one case three yards, from the ileo-cæcal valve upwards, were irregularly contracted, while above this the gut was dilated. There was slight congestion of the peritoneum, but no adhesion, except where parts had been divided, and no fluid in Douglas's pouch. There was no occlusion of the lumen of the gut, for the cæcum was also distended, and flatus had passed from the anus freely during the course of the case. The condition of the intestine, however, must have added greatly to the labour of its muscular wall, and Mr. Meredith informs me that the patient died with all the symptoms of obstruction, except that flatus passed freely from the anus. I have also repeatedly seen-post mortem-the large gut, throughout or in part, much smaller than normal. This I attributed at first to the pressure of tumours, but I have found a similar condition in cases in which the tumours removed had been so small that this explanation could not hold good.

In cases of obstruction of the intestines, whether arising from want of power in the gut or from mechanical causes, the rapidity with which symptoms develop depends mainly on the condition of the intestine and the amount of gas and secretions which are developed in it. As a rule, unless some other complication exist, there is no untoward symptom immediately after the operation. When gases begin to form the semi-paralysed bowel yields at once and painlessly, or with slight colicky pains. The first unfavorable symptom, therefore, may be abdominal distension, occurring about the time when flatus ought to have begun to pass. But if the obstructive condition continue it soon becomes evident that large quantities of watery secretions are being produced by the glands opening into the intestine above the obstructed point. This development of fluid appears to be brought about by a reflex mechanism in the same way as the salivary secretion is often induced before the act of vomiting. Gaseous and fluid matters accumulate in the intestine and distend it till the stomach is no longer able to propel its contents into the duodenum. If food be then given it is imme- 
diately rejected with more or less of watery gastric secretions. Sooner or later bilious fluid and the intestinal contents pass upwards into the stomach. Constant vomiting supervenes and an enormous quantity of fluid may be discharged from the mouth, its colour changing as the case progresses from that of the gastric juice to yellow, green, and brown, and finally to a very dark hue, "coffeeground" or "black" vomit. I have only seen stercoraceous vomiting once in at least five cases of death from obstructed intestine after abdominal section.

As the intestine becomes distended the branches of the mesenteric arteries are subjected to increasing pressure, and this adds greatly to the vascular tension already produced by the inflammatory fever. The heart beats at first more strongly in its endeavours to overcome the resistance thus induced, but the extra work, the depressing effects of the vomiting, and the wasting of elaborate secretions, soon diminish the general and especially the cardiac power, and the pulse becomes small, fast, and feeble.

Thus far the results of retention of flatus and the constant results of the operation are developed side by side, the temperature not being much, if at all, deflected from the course which it usually follows in a case which is doing well. In such a case the temperature reaches its highest point on the second or third evening after the operation and then gradually falls to normal at the end of about a week; so that if the retention of flatus be the only complication, unless the development of gases begin unusually early, the temperature is falling, though perhaps not steadily, when the signs of retention of flatus are becoming prominent. But as exhaustion increases, the inflammation depending on the healing of the parts divided at the operation becomes exacerbated from enfeeblement of resisting power in the tissues, and fever increases. The retention of flatus and the inflammation react harmfully on each other. The bowel, distended to its utmost limits, or to the utmost capacity of the abdominal cavity, becomes quiescent, and all the parts divided 
at the operation adhere to adjacent peritoneal surfaces. Finally, inflammation spreads from the wounded parts to the general peritoneum, and as this advances the patient rapidly dies of exhaustion and pyrexia, generally about the fifth day, the symptoms of obstruction having become prominent on the second or third day after operation.

These cases of obstruction are characterised by a remarkable clearness of mental power till within a very short period before death. This is one of the most important signs by which we may distinguish symptoms of obstruction from those of septic origin. Another is the condition of the urine. This secretion is, so far as my experience goes, only partially suppressed a few hours before death in cases of obstruction, and albuminuria does not occur. I have always found a dark colour produced on contact of the urine with strong nitric acid from the presence of excess of indican.' The colour becomes more intense as the severity of the symptoms increases, and in cases which recover the reaction becomes gradually less marked as the other symptoms of obstruction pass off.

In these cases the post-mortem appearances in the peritoneum may be normal or those of commencing inflammation. Divided surfaces are usually adherent to adjacent portions of peritoneum, and adjacent coils of intestine may be glued together, but, as a rule, there is no visible lymph formation and no fluid in Douglas's pouch. The intestines are immensely distended by gases and liquids, and a mechanical obstruction of the bowel may or may not be found.

The sequence of events detailed above as following mechanical obstruction of the gut, and the accompanying symptoms, occur in all cases in which death results with retention of flatus after abdominal section. But the symptoms are modified by the nature of the cause of the retention and by the accompanying circumstances.

I now turn to the effects produced by the exudation of blood and serum or of other matters into the peritoneal 1 'Parkes on the Urine,' p. 196. 
cavity. The exudation of blood and serum in very variable amount is a constant sequence of operations which involve section of the peritoneum. If a case do well, the exuded fluid is quickly absorbed; but if an abdomen be closed when there is much exudation or little absorbing power, or when the tissues are already saturated with serum, as in excessive odema, the fluid in the peritoneum-even though quite aseptic-may act as a foreign body. And irritating or septic matter invariably does so. Under such circumstances, complete muscular paralysis of the gut is produced, with all the symptoms of obstructed intestine. Distension of the bowel follows, and fever runs high. These signs come together, but the high temperature and rapid pulse which concur with the distension are not due to inflammation but to irritation, and mainly to tension of fluid in a closed sac. That this is so is shown by the facts that the temperature in these cases at once rises when the abdomen begins to distend; but that if a drainage-tube be then passed into the bottom of the pelvis, and the fluid be thereby removed, the temperature immediately falls; and that in exactly similar cases, if Keith's method of drainage be employed from the time of the operation, there will in all probability be little or no fever, for the drainage prevents tension and reduces to a minimum the strain on the absorptive powers of the peritoneum. I have even seen very offensive pus spilt over the peritoneum, and by careful cleansing and the use of the drainage-tube all ill-effects were prevented.

If, on the other hand, the fluid be not removed by drainage, nor by absorption, symptoms of obstruction and of peritonitis rapidly develop. The paralysis of the intestine, the tension and continuous irritation in the peritoneum, with the high temperature and increasing exhaustion, quickly induce general peritonitis, and the patient dies of this disease usually before the fourth day after operation.

In these cases also (unless death be from shock) the 
mind usually remains clear, though it may wander from high temperature. The urine may not be suppressed until great exhaustion is induced; but albuminuria and suppression of urine may occur, for the strain on the kidneys is great both from vascular tension and from the presence of products of inflammation which must be excreted if any attempt at repair be made.

At the necropsy in this class of cases signs of general peritonitis, or at least of commencing general peritonitis, are found, with much fluid in Douglas's pouch; but masses of lymph and centres of pus formation do not necessarily exist, and when they do, they indicate that some matter more irritating than simple discharges from healthy surfaces has been present in the peritoneum.

If $I$ interpret the signs and symptoms aright, general peritonitis-in the absence of septic influences-is one of the rarest of the primary complications of operations involving division of the peritoneum. Peritonitis must of course occur whenever the peritoneum is divided and does not heal by first intension. But it appears to me that the inflammation which causes fever after abdominal section is mainly in the divided parts outside this membrane, and that the peritonitis which occurs is accurately comparable to inflammation of the skin edges in a superficial wound. When a surgeon speaks of the skin being inflamed in such a case, he means a local inflammation, and when he wishes to imply a more extensive dermatitis, he uses a different word-erysipelas or erythemaor at least a qualifying adjective. So in the case of the peritoneum it is necessary to draw a clear distinction between local traumatic peritonitis and the diffuse inflammation of that membrane which may occur after an abdominal section.

It is of course possible that the necessary exposure and manipulation might cause inflammation of the peritoneum generally; but the course of the great majority of cases indicates that the discharges into the peritoneal cavity are very rapidly absorbed, and that therefore in 
these cases a sufficient surface of peritoneum to effect this purpose remains healthy. When it is necessary to reopen an abdomen a few days after an operation (but of course before the patient is moribund), or when a second operation is required after a prolonged interval, the conditions found also point to the absence of primary general peritonitis. And all I have seen of post-mortem examinations after abdominal section, when considered in connection with the clinical history of the cases, seems to corroborate the view that general peritonitis is rarely produced as a direct result of the operation, but that the peritoneum may be subjected to any necessary treatment without more than a local peritonitis being produced at the divided or severely bruised and adjacent parts. General peritonitis may of course exist when an operation is performed ; and few cases die in consequence of abdominal section (except of shock or hæmorrhage) without evidence of its commencement at least being found at the necropsy. But I have convinced myself, and have endeavoured in this paper to show that general peritonitis after abdominal section is usually, if not always, a secondary effect of the operation, though often it may be the actual cause of death.

Septic peritonitis is a disease arising from a specific influence and communicable from case to case. $\mathrm{Mr}$. Lawson Tait has written a description of this disease following abdominal section. It runs as follows :- "When from some reason or another the patients begin to do badly, the first indication is an altered expression of the face. I am unable to describe this change of countenance, but I learned to recognise it only too well in the old days, when from the use of the clamp my mortality ran high. Associated with this changed expression is a rapidly increasing abdominal distension, speedily followed by vomiting. At first the vomited matter is simply the fluid the patient has swallowed, but soon it becomes tinged with bile. Later on, should the patient grow worse, the vomited matter becomes entirely bilious, and towards the 
end it gets quite black and characterised by those features to which the name of 'coffee-ground' vomiting has been aptly given. In those instances where death followed the use of the clamp, the phenomena always began on the second or third day, the patient dying on the fourth or fifth ; and when once these fatal symptoms had become fairly established, nothing I ever did could arrest them."'l

These symptoms exactly correspond with those which I have described as due to obstruction, but with the addition of an indescribable change of countenance. The commencement of the symptoms on the second or third day after the operation was due to the origin of the disease. Mr. Tait attributes "the fatal result in such cases to the presence of a minute aperture in the wound at the point where the pedicle was embraced through which the discharges from the ulcerated surface under the clamp penetrated into the abdominal cavity.", The date of commencement of the signs of fatal mischief must therefore have corresponded with the date at which the discharges became septic. The similarity in the symptoms to those of obstruction is due to the fact that septic peritonitis at once and completely paralyses the muscular power of the intestine, and so gives rise to what may be called paralytic obstruction in its most marked form. But to complete the account of the symptoms of septic peritonitis, it is necessary to add those due to the effects produced by the poison on the organism generally. The most definite of these are the constant presence of delirium or mental vacuity, the strong tendency to albuminuria, and suppression of urine, the rapid emaciation of the patient, and the marked rise of temperature which precedes death. In fact, in cases of septic peritonitis there are the signs of a triple pathological state, of peritonitis, of bloodpoisoning, and of paralytic obstruction of the bowels, the intestinal symptoms being entirely secondary to the paralysis caused by the septic inflammation of the peritoneum.

1 Tait's ' Diseases of the Ovaries,' 4th edit., p. 308.

8 Ibid. 
The port-mortem appearances are those of intense general peritonitis, usually of a suppurative character.

From a consideration, therefore, of all the circumstances which may attend the operation of opening the abdominal cavity, I conclude that there are two conditions to which retention of flatus may be due in these cases ;-first, mechanical obstruction to its passage down the gut; second, paralysis of the muscular power of the intestine; and that this last may arise from over-stimulation during operation, from continued stimulation after operation of the peritoneum covering the gut, or from general septic peritonitis. It is usually impossible to distinguish (except by opening the abdomen) between symptoms due to mechanical obstruction and those due to paralysis of the intestine from over-stimulation during operation ; but the several causes of paralysis and the modes of death therefrom are distinct in their origin, in the course of their symptoms, and in their post-mortem appearances. In any case the intestinal distension is not in itself a disease but merely a result of mischief, though it may in turn become an originating or an aggravating cause of inflammation.

In the management of the intestine in relation to an abdominal section the surgeon must use every endeavour to conserve its power and to decrease its work. To this end it is necessary to thoroughly empty the bowels of all scybalous masses or other accumulations before operation; and perhaps the best means of doing so is to give a dose of castor-oil with ten grains of Dover's powder. These medicines, or some other form of purgative, should be given three or four days before the operation and repeated two or three days later, an enema being administered on the morning of the operation. If time permit too much attention cannot be paid to getting the bowels into a healthy condition. Masses of fæces may become more or less impacted in the intestine either from costive habits or from the effects of disease; and the preparatory administration of purgative remedies, or the manipulation 
during operation may merely dislodge these scybala from positions in which they have been hitherto harmless. Such scybala are not infrequently passed per anum after operation, often with severe pain and alarming fever from disturbance of partially healed tissues. But in the condition of the intestine which follows operation it may be unable to expel these masses, and they may become the cause of complete or partial obstruction and of the death of the patient. I have made one post-mortem examination, in which a scybalous mass was found to have been forced into a constricted portion of the descending colon, where it produced an almost complete obstruction. The constricted part was about four inches long and only admitted one finger. It contained a series of five or six little hard pieces of fæces, which fitted it tightly, and there were large hard masses in the distended bowel above. In the absence of $\mathrm{Mr}$. Thornton I had charge of this patient after the operation, and when she showed symptoms of obstruction I endeavoured to move the bowels by repeated and increasing doses of saline purgatives, but I only succeeded in getting flatus to pass with very temporary relief, and the patient died on the fifth day after operation with all the symptoms of obstruction which I have described. Evidently the constricted condition of the colon which prevented the masses of fæces from coming into the rectum after operation had also caused the accumulation of these masses and had prevented their removal by the preliminary purgation.

During an operation all unnecessary handling and exposure of the peritoneum should be avoided, and every attention should be paid to leaving the abdominal contents as nearly as may be in their natural condition. Every bleeding point should be secured, and Keith's method of drainage should be used whenever a doubt exists as to the power of the peritoneum to absorb the discharges. Of course, too much trouble cannot be taken to prevent contact of the tissues with everything that could possibly convey sepsis to the blood, but it must be remembered 
that carbolic acid and Lister's spray producer have a stimulating and chilling effect on the nerves and muscles of the intestine which can only be harmful.

After operation, intestinal rest is obviously desirable on physiological grounds. When the sickness caused by the anæsthetic has ceased, only the most easily digestible food should be given, and if there be any sign of gastric or intestinal irritability, nutrition should be effected entirely by the rectum. Unless under special circumstances contraindicating the use of opium, the best and most rational treatment is to give this drug, or morphia, in sufficient quantity to allay pain. During severe operations there may be much interference with the bowel and mesentery, and laceration of these parts in the separation of adhesions. In such cases, when all bleeding points have been secured, there must of necessity be much temporary disturbance of the supply of blood to the affected and the neighbouring parts of the intestine. But interference with the blood supply causes spasmodic contraction, muscular irritability, or paralysis of the affected parts of the gut in accordance with the condition of the blood in the capillaries, and the amount of other stimulation to which the intestine has been subjected. Under such circumstances, to keep the whole intestine as quiet as possible for a time would seem to be one of the plainest indications. Opium is our best agent for this purpose, and in the absence of complications opiates do not paralyse the bowel, but under their influence flatus passes freely and the abdomen remains soft and flat. The intestinal movements are certainly never abolished by opium in any quantity short of a lethal dose. Peristalsis seems to be of the same nature as the cardiac and respiratory movements in that after a period of rest an imperative necessity arises for its recurrence; but the movements of the intestine are much more irregular than are those of the heart and respiration. Opium prolongs the intervals between the peristaltic waves, and, in an embarrassed condition of the bowel, the prolonged period of rest thus 
induced, and the consequent greater accumulation of nervous energy in the automatic motor plexus of the gut, are followed by a stronger, steadier, and more effectual peristaltic contraction. Opium, therefore, after an abdominal section, quiets the action of the gut, secures as much as possible the intestinal rest which is so greatly needed, and tends to prevent those irregular, fruitless, and exhausting efforts which are so undesirable at this time. And if the bowels have been properly emptied beforehand there is no necessity for exertion on their part for some days at least after an operation. Opium may undoubtedly do harm in some cases and in some conditions, but there is abundant evidence of its beneficial action, after abdominal section, if given intelligently while its general and special effects are carefully watched.

If, however, retention of flatus, followed by distension or vomiting, come on in the first few days after an abdominal section, and if, from a careful consideration of all the facts of the case, a simple obstruction in the intestine be diagnosed, its cure may be attempted by rest and sedatives, by stimulating the muscular activity of the intestine, or by reopening the abdomen and giving mechanical relief. But it is well known that the treatment of obstruction of the bowels by sedatives acts slowly, and we have seen that during convalescence from an abdominal section, when once the proper balance is lost between the strength of the bowel and the work to be done by it, when regurgitation in the intestine is taking place, it is only too likely that a train of events leading to a rapidly fatal issue has been started. When symptoms of simple obstruction occur, therefore, so long as flatus passes from the rectum from time to time, and vomiting is not constant, it is possible that by the steady administration of sedatives, and by withholding all food from the stomach, the bowel may so far recover as to be able to remove the obstruction. But if flatus be retained and vomiting or distension come on while sedative treatment is in active operation; if the patient be evidently getting worse, and 
especially if the pulse-rate be rising, and symptoms of exhaustion be marked, the time for dependence on the effect of sedatives is past. The progress of the disease towards a fatal issue will now be more rapid than a cure by sedatives can be. At any moment the resisting power in the peritoneum may become so lowered that general peritonitis is inevitable, and it is of no use to have the obstruction removed after such a degree of depression is reached, for the very nature of the cause of the peritonitis in these cases-the progressive debility-renders that disease when thus induced almost certainly fatal. There is therefore not a moment to lose. The only hope is to rapidly clear the bowels, or at least to overcome the obstructive condition before general peritonitis is excited.

This ought to be attempted in the first instance by purgatives, the rectum being of course emptied by enema if it contain any fæces. Mr. Lawson Tait says that in his practice vomiting and distension are now extremely rare, and for their treatment he recommends " a small dose of sulphate of magnesia, thirty or forty grains in tepid water, repeated every hour or every other hour until the bowels have moved, or two and a half grains of calomel given every three or four hours until a similar effect is produced."1 This treatment is excellent in all cases in which retention of flatus is caused partly by some slight and easily removable mechanical obstruction to the passage of the contents of the bowel, but chiefly by weakness of the gut from exhaustion of the motor nervous centre in its wall. In such cases a timely stimulation of the intestine may produce a most beneficial effect. I have seen several cases in which distension and romiting came on during convalescence from abdominal section, and in some of which death seemed inevitable under sedative treatment, but which immediately improved and steadily recovered after an action of the bowels had been induced by small doses of saline purgatives. Of course it is im1 Tait's 'Diseases of the Ovaries,' 4th edit., p. 314. 
possible to tell what the exact cause of the difficulty in these cases was, but there evidently must have been either a simple exhaustion of the gut or some condition sufficient to seriously obstruct the passage of the weakened intestine, but so insignificant that a very slight stimulation of the peristalsis completely and permanently removed it. Such cases are, I believe, not rare, and the symptoms and ultimate result, though due to weakness of the gut rather than to obstruction, are, if persistent, exactly the same as those of mechanical opposition to the passage of the intestinal contents.

And in these cases the form of purgative is of minor importance, and it matters not how soon after operation it is given,-as a rule, the sooner the better,-provided the treatment be effectual in removing the obstructive condition. This proviso is of the utmost importance; for if for any reason the object of the peristalsis be not accomplished, the extra exertion is necessarily followed by a more decided exhaustion; and the condition of the patient becomes worse than before. There is therefore in these cases good reason for giving a purgative soon, if abdominal distension or vomiting commence. For, if given early, the medicine will act on a comparatively healthy gut and not on one that has lost all tone by excessive stretching; it will act before general peritonitis has set in, and in cases in which this line of treatment fails to give mechanical relief, it will in a short time, by stimulating the secretions intensify the symptoms, and so clinch the diagnosis that the surgeon must at once consider the question of reopening the abdomen in the expectation of finding a definite mechanical obstruction, and being able to relieve it before exhaustion becomes excessive, a course which he might not otherwise feel justified in adopting so soon, but which is now his only hope.

If on the other hand a free action of the bowels can be secured before general peritonitis comes on, and if the obstruction do not return, the difficulty to the circulation is removed, and the powers of nature which have thus 
far borne up the patient are strengthened by lessening of work and by nourishment which can again be taken. The good effects thus produced more than counterbalance any ills which may result from disturbance of the bealing processes, or from separation of recent adhesions, for any fresh discharges which may be thrown out are speedily absorbed, and the patient may be expected to do well.

But when it is diagnosed that retention of flatus is due not to a mechanical cause but to paralysis of the intestine from irritation of the peritoneum by discharges, then the first thing to be done is to remove the discharges by drainage. It is, however, very much better that, if this be likely to become necessary, a drainage-tube should be inserted at the time of operation. To put one in afterwards is difficult, and must be looked upon as a curative measure of doubtful utility, whereas drainage from the first is a preventive proceeding of tried efficiency. An exit for the discharges having been made, if any mechanical obstruction in the gut be also supposed to exist, if vomiting or distension occur, unless there be marked evidence of peritonitis, a purgative should be given. The unloading of the bowel favours the progress to resolution of the local inflammation in the divided parts, as purgation aids the healing of any wound at a distance from the intestine. And unless general or extensive peritonitis or a doubtful state of vitality in some part of the bowel also exist, no harm will result from the movement of the coils of intestine on each other. This will rather do good by aiding the progress of any free fluid in the peritoneum to the bottom of Douglas's pouch, whence it may be removed by the drainage-tube. Moreover, if adhesions be torn any fresh serum which may be thrown out is in this way at once got rid of. And the fact that adhesions are torn shows them to have formed in such positions that they may have been the cause of retention of flatus, or that they might afterwards have become sources of inconvenience to the bowel, possibly of danger to the patient.

If irritation from within the peritoneal cavity be alone vol. LXXI. 
believed to exist, then the treatment for peritonitis should be employed, and as the stimulation of the intestine is in these cases severe and prolonged the necessity for rest is afterwards great.

When diffuse peritonitis (not septic) is present at the time of operation, or is produced by the operation, or by the irritation of retained discharges afterwards, the treatment should be very different from that of paralytic or obstructive retention of flatus. In the cases now under consideration we have to deal with an inflammation arising from an injury. It is of the nature of a traumatic inflammation, and therefore has a tendency to run a definite course ending in resolution, provided the cause be not persistent, and the patient's strength can be maintained. Tension and irritation must therefore be removed by drainage in these cases also. The most absolute rest possible should be secured to the bowel by the administration of opiates, and this is of course the more necessary if doubt exist as to the vitality of any portion of the gut.

The administration of "a rapidly acting purgative" has been recommended by Mr. Lawson Tait "on the slightest indication of peritonitis after ovariotomy," and Mr. Tait says that under this treatment the peritonitis "disappears." It is not to be denied that a rapidly acting purgative may, possibly, cut short or cure a peritonitis in its initial stage, as it is certain that free purgation does sometines cure or modify other inflammations. But I have endeavoured in this paper to show that the abnormal conditions to which the intestine is subjected during an abdominal section constitute a sufficient stimulation to exhaust the energy of Auerbach's plexus of nerves; and that in the resulting condition of the bowel may be found the explanation of many symptoms, and of not a few deaths, hitherto unexplained, or erroneously attributed to inflammation and other causes. And I have endeavoured to show how, when this condition gives rise to retention of flatus the administration of a purgative may

1 ، Brit. Med. Journal,' May 15th, 1886, p. 921. 
be followed by the happiest results, preventing general peritonitis by removing its cause. The combination of symptoms which may be induced by this condition of the bowel is nearly similar to that caused by extensive peritonitis; but there are usually sufficient grounds for making a differential diagnosis, and it certainly does not seem to have been satisfactorily proved that in cases of general or extensive peritonitis following abdominal section, the administration of a purgative is advisable. Indeed, this treatment seems exceedingly irrational, for it must give rise to much friction of inflamed serous surfaces, and the risk of rupture, if the bowel be damaged, must be great. But this treatment is also impracticable when severe peritonitis is established. Purgatives act by stimulating the nervous centres in the wall of the gut, or the muscular and secretory tissues directly. These, however, may be already completely paralysed by the peritonitis so that the intestine cannot by any means be stimulated to evacuate its contents. ${ }^{1}$ Dr. Keith, of Edinburgh, has told me, and I have his permission to publish the statement, that "in some cases of septic peritonitis, you may give the patient what you will, the bowels won't move." In cases of general peritonitis, therefore, the administration of purgatives can only lead to fretting of the bowel and of the peritoneum, to secretion of more intestinal fluid, to a more profound intestinal paralysis, and to aggravation of symptoms and of danger. Simple general peritonitis and septic peritonitis can both be prevented with much certainty. If, however, this end be not attained the prognosis is extremely grave; but unless some other dangerous complication exist, rest, suitable nourishment, and the removal of irritation by drainage are the rational means of treatment.

Many of the facts and theories which I have brought forward in this paper have been suggested by the postmortem appearances which I have found at the necropsies of cases of abdominal section at the Samaritan Free

1 Stirling's translation of Landois' 'Physiology,' 2ud edit., p. 340. 
Hospital; but I have to express my thanks to $\mathrm{Mr}$. Knowsley Thornton for permission to publish many observations which I have made while assisting in his practice, $\bullet$ and it gives me much pleasure to have the opportunity of saying that to my good fortune in being the colleague and assistant of this master of his craft, I owe my practical knowledge of the art of surgery as specially applicable to the region of the abdomen. I would also add that I am indebted for a great deal of information on this subject to the friendship of the Keiths of Edinburgh, who, when opportunity offered, have given me every facility for seeing their practice, and from whose conversation I have learned much while enjoying their hospitality.

(For report of the discussion on this paper, see 'Proceedings of the Royal Medical and Chirurgical Society,' New Series, vol. ii, p. 29.) 